\title{
Enhanced Fusion Mechanisms Towards Synthesizing Superheavy Elements
}

\author{
Bolivar N. and Vasilev I.T.
}

\begin{abstract}
In nature all of the heavy elements are produced by nuclear fusion reactions, mostly in supernova explosions and neutron star collisions, so, this is to date the only known and proven mechanism to produce heavy elements in usable quantities. In this work we approach a difficult challenge, namely, the possibility of fusion of heavy elements, taking as a test case the heaviest observationally stable element $-{ }^{238} \mathrm{U}$, showing that it is feasible, at least in principle with the help of existing technologies. The main idea behind is to show that fusion of lighter - than $z=184$ - nuclei is conceptually viable examining the tunnel effect assisted by an auxiliary field that will produce a Sauter like effect, and this is the pathway to explore the synthesis of elements higher than $z=118$. The production of theoretical untested elements like Unoctquadium-184 or close $Z$ species could open a new chapter in the physics of super-heavy elements, and leads to a deeper understanding of nuclear decay channels and stability conditions. Nuclear fusion of heavy elements will open the breach to produce neutron rich elements, so we may obtain a deep insight into the physics of the island of stability. This work will review basic aspects of fusion physics related to the assisted fusion mechanism. An enhanced fusion perspective is found generalizing the work of [1] to space dependent fields and the cases of ${ }^{2} \mathbf{H},{ }^{106} \mathrm{Pd}$ and ${ }^{238} \mathrm{U}$ are presented for several test fields. A final section reviewing laser confinement fusion actual experiments capable of achieving the required energies is also reported.
\end{abstract}

Index Terms-Assisted Fusion, Confinement Fusion, Sauter Effect, Heavy Elements.

\section{INTRODUCTION}

A nuclear fusion reaction is the one that results in a nuclide with a mass number greater than that of each of the initial nuclides. Fusion reactions of astrophysical interest are the proton-proton cycle, which takes place on the Sun.

$$
4 p \rightarrow{ }^{4} \mathrm{He}+2 e^{-}+2 \bar{v}+26,732 \mathrm{MeV} .
$$

Another present cycle is $\mathrm{CNO}$, and the triple-alpha process:

$$
{ }^{4} \mathrm{He}+{ }^{4} \mathrm{He} \rightarrow{ }^{8} \mathrm{Be}-91.8 \mathrm{keV}
$$

. It is worth noting that fusion reactions cease to be exothermic when the resulting element is heavier than the ${ }^{62} \mathrm{Ni}$. Thus, the processes of nucleosynthesis in stars through fusion reactions produce only relatively light elements, explaining the abundance of elements around ${ }^{56} \mathrm{Fe}$. Heavier elements are produced by other processes, such as neutron capture.

Assume that a beam of particles strikes a thin target and that we want to know the number of reactions of a certain type

DOI: http://dx.doi.org/10.24018/ejers.2021.6.1.2242

Published on January 06, 2021.

N. B. is with the Escuela de Física, Facultad de Ciencias, Universidad Central de Venezuela, Venezuela. (e-mail: nelson.bolivar@ciens.ucv.ve).

I. V. is with the Ingenieering Department, Technical University of Sofia, Bulgaria. (e-mail: ivaylo_vasilev@tu-sofia.bg). produced per unit of time as a result of the interaction of the particles with the target. For this reason it is useful to assign to each target nucleus an area $\sigma$ such that we can imagine that only when the incident particle reaches this area the reaction takes place. The area $\sigma$, called the effective cross-section, is the effective surface area that the nucleus presents to the beam in relation to a given process. The usual unit of the effective cross-section is the barn $\left(1 \mathrm{~b}=10^{-24} \mathrm{~cm}^{2}\right)$. Assume that the incident particle current is $I_{a}$ particles per unit time and that the target presents to the beam $N$ nuclei per unit area. If the projectiles appear at the rate of $R_{b}$ particles per unit time, then the reaction effective cross-section is

$$
\sigma=\frac{R_{b}}{I_{a} N}
$$

The effective cross-section can be much larger or smaller than the geometric area of the disk that the target nucleus presents to the incident beam, depending on the energy and direction of the emitted particle. As a matter of fact and as an extreme case, for Coulomb interactions it is infinite and only its differential form in terms of the scattering relevant parameters is going to be finite. Under experimental conditions, a small fraction $d R_{b}$ of the outgoing particles are detected within a solid angle $d \Omega$. In general, the outgoing particles will not be emitted uniformly in all directions, but will have an angular distribution that depends on the angles $\theta$ and $\phi$ that determine the orientation of the direction of the salient particles with respect to that of the incident particles. Let the angular distribution be $D(\theta, \phi)$, then $d R_{b}=D(\theta, \phi) d \Omega / 4 \pi$. Therefore,

$$
\frac{d \sigma}{d \Omega}=\frac{D(\theta, \phi)}{4 \pi I_{a} N}
$$

The quantity $\frac{d \sigma}{d \Omega}$ is called the differential cross-section.

In many applications it is not only interesting to know the probability of finding outgoing particles of a certain type and at a certain angle, but also to have their energy within a $d E$ range around a particular $E$ value. The differential double effective cross-section is then defined $d^{2} \sigma / d E d \Omega$. In the literature this additional dependence on energy is often not explicitly mentioned. Usually the effective sections are plotted as $d \sigma / d \Omega v-\theta$ for a defined energy end state.

There is a numbers of protons or neutrons that give a particular stability to the formed nucleus. These "magic numbers" are experimentally found to be $2,8,20,28,50,82$ and 126 . These numbers seems to indicate that nucleons move at an average potential in much the same way as electrons do around the nucleus.

There are only certain combinations of neutrons and protons, which form stable nuclei. The neutrons stabilize the 
nucleus, which roughly speaking helps to compensate for the electrical repulsion between the protons. As a result, as the number of protons increases, the relation neutrons/protons also increases in order to form a stable nucleus. Neutrons are fermions, which obey the Pauli exclusion principle, it basically dictates that each energy level is characterized by a certain group of quantum numbers; energy, spin, among others. A single particle can occupy a level, leaving no room for another, as a consequence fermions do not condensate. It is worth mentioning that the very fermionic nature of nucleons allows for the formation of shells, nevertheless pairing for stability could seem contradictory to this end for even numbers of nucleotide pairing of nucleons that are "close" in a shell is possible. This bosonization in cooper pair like particles, do not imply these pseudoparticles are excluded from the Pauli's exclusion principle, as is noted in [2], [3] and references therein. In these works related to BCS and BEC (Bose Einstein Condensate) theory, two Cooper Pairs show a decrease in the average binding energy at expenses of the increasing number of pairs. This due to the decrease of the number of available states to form pairs.

In the case of BEC the impenetrability, i.e. inability to occupy the same point in a space/lattice site, could have two possible origins. For example, for liquid Helium it emerges from the repulsive pair interaction, while for composite bosons it arises from the Pauli exclusion principle acting on to their fermionic constituents.

For instance, in [2] they show that the hard-core constraint imposes an upper bound on the maximal possible degree of Bose-Einstein condensation. In complete analogy to Pauli's exclusion principle for fermions, this bound is universal for any lattice type and for any underlying Hamiltonian. Clearly, the Pauli exclusion principle for the fermionic constituents lead to qualitatively similar bounds on the degree of BEC of composite bosons. Nevertheless, the degree of internal entanglement between the constituents will affect the impenetrability.

Given that the separation in energy between the levels that form a layer is much less than that existing between two levels of different layers, adding an electron to an atom of an inert gas implies a much greater energy cost than that related to adding an electron to an atom whose last layer is not filled. This is why the atoms of an inert gas are particularly stable.

It should be noted that, unlike the strong central Coulombic potential in atoms, there is, in principle, no such thing in nuclei. However, we can picture such a potential created by the action of all nucleons. In other words, the motion of these independent particles can be thought to be governed by a mean potential. Let's consider a nucleon near the center of the nucleus. For reasons of symmetry, the net force on it should be zero and therefore it has to be:

$$
\left(\frac{\partial V(r)}{\partial r}\right)_{r=0}=0
$$

Since the binding forces increase as you approach the nucleus "surface", the derivative is positive inside this limit and can be considered to vanish outside. The Woods-Saxon potential represents quite well this characteristics, it is given by

$$
V(r)=-V_{0}\left(1+e^{\frac{r-R_{0}}{a}}\right)^{-1}
$$

with $R_{0}=r_{0} A^{1 / 3}$

Since the eigenfunctions of this potential cannot be given in a closed form, it is usual to follow two qualitative approaches, namely use square well and harmonic oscillator approximations. Both eigenfunctions are not correctly bounded, but works very well as test functions. Let's consider the harmonic oscillator energies, these are equidistant levels

$$
E=\frac{h}{2 \pi} \omega\left(N+\frac{3}{2}\right)-V_{0}
$$

where $N=2(n-1)+l$, being $N$ the quantum number of the oscillator, $n$ the radial quantum number, $l$ the angular momentum and $\omega$ the frequency of the oscillator. The degeneration of levels is giv en by $D(N)=1 / 2(N+1)(N+2)$. This accidental degeneration of the harmonic oscillator is removed by the square well potential. The energies sought, i.e. those corresponding to the Woods-Saxon potential, will lie between the limits given by these two potentials.

The scheme of levels with nucleons is complemented if we observe that according to the Pauli exclusion principle, $D(N)$ protons and $D(N)$ neutrons can be placed within each layer, in a group of degenerated states with equal $\mathrm{N}$ of the oscillator. This means that both potentials reproduce the magic numbers, 2, 8 and 20 .

Qualitatively the shell model adds a spin-orbit interaction to the square potential well. The interaction between nucleons becomes now spin-dependent, along the same lines as the atomic spin- orbit interaction, which slightly modifies the energy levels by adding a spin-orbit term to the potential.

In the atomic case, a term of the form $f(r) \vec{L} \cdot \vec{S}$ results in the rupture of the degeneration, with the appearance of levels $j=l \pm 1 / 2$.

Here, the levels are characterized in the coupled scheme by the quantum numbers $n, l$ and $j$ and it can be shown that the separation of the doubly degenerated levels for $f(r)$ constant is:

$$
\Delta E(l)=2 l+1
$$

This equation also shows that the gap increases as the value of $l$ increases, as shown in Fig. 1.

\section{OVERVIEW OF THE FUSION OF NEW ElEMENTS - THE ${ }^{238} \mathrm{U}$ PERSPECTIVES}

During the last century the formation of new elements became an exciting research area, when Fermi and his coworkers bombed a neutron uranium target, this led to the formation of new elements beyond $Z=92$ through the capture of neutrons with subsequent $\beta$-decay. This method achieved remarkable success in the production of heavy elements up to $Z=100$, where the spontaneous emission ended the periodic table. This limit gave birth to a new era of heavy and super-heavy element formation with heavy ion beams. Beams ranging from alpha to ${ }^{86} \mathrm{Kr}$ particles and targets as heavy as 


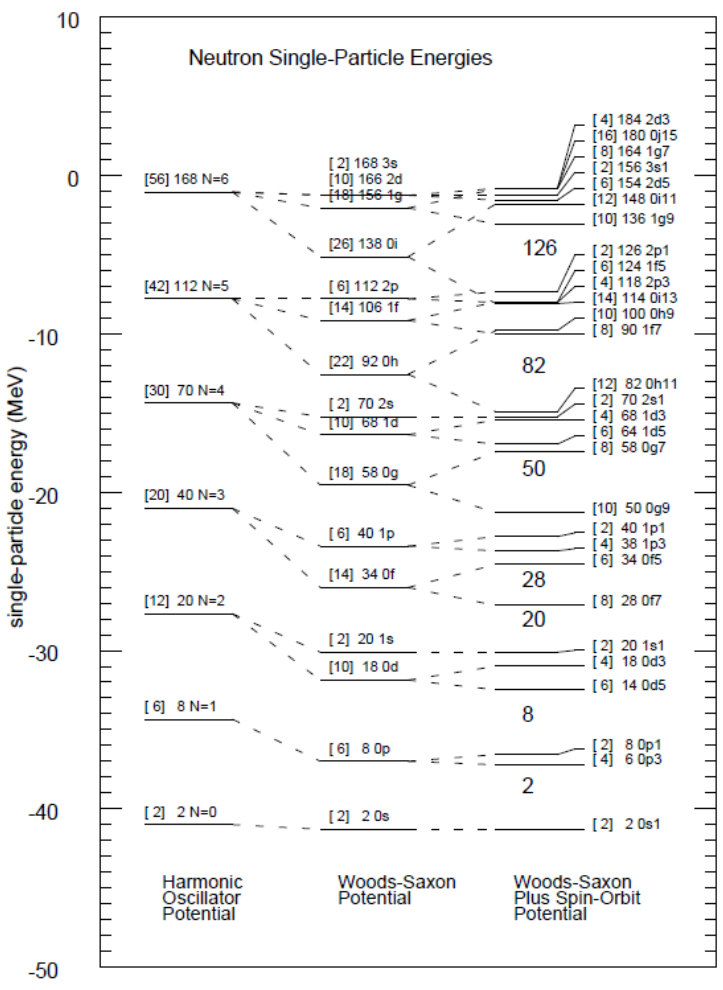

Fig. 1. Shell model. Energies of a single neutron. The degeneracies are broken from the Wood Saxon and Harmonic Oscillator potentials in the presence of spin orbit interaction. The magic numbers are nicely reproduced.

${ }^{208} \mathrm{~Pb}$ and heavier radioactive cores have been used to form elements with a maximum nuclear charge of $Z=118$.

The production of elements with $Z>119$ presents several technical problems if you follow the conventional method of production of under 118 , where ions of ${ }^{48} \mathrm{Ca}$ are irradiated over heavier transuranic cores than berkelium. On the one hand, the use of heavier projectiles than ${ }^{48} \mathrm{Ca}$, which have an excess of neutrons, are not nuclei doubly magical, which means the odds for the formation of the composite nucleus decrease drastically.

Mainly, two types of fusion processes are known for the synthesis of Super Heavy Elements, i.e. above and below the Coulomb barrier. The first uses transuranic targets and involves the formation of compound nuclei with excitation energy large enough to evaporate nucleons before reaching the ground state. The latter approach is intended to use a combination of projectile and closed-shell target to form the composite cores with low excitation energy, so that you can only evaporate $\sim 1$ neutron. In this fusion process, the increased cross-section is observed at an incident energy below the interaction barrier.[4] [5]

The first obstacle to achieve fusion is the Coulomb repulsion between the nuclei, because of their proximity the energy associated with this barrier is too large and for two nuclei is written as,

$$
V \approx 1.638 \times 10^{-13} \frac{Z_{1} Z_{2}}{A_{1}^{1 / 3}+A_{2}^{1 / 3}} \mathrm{MeV}
$$

where $A_{1,2}$ are the atomic masses of each species, $Z_{1,2}$ are

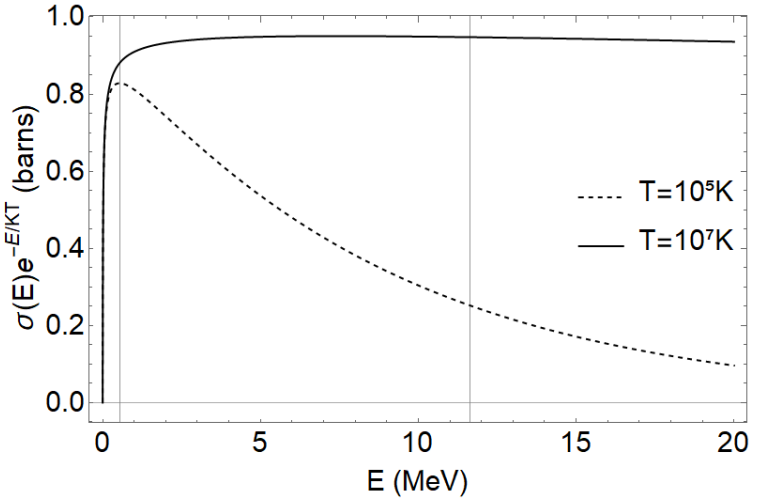

Fig. 2. Cross section weighted with a Maxwell distribution for a $U^{238}$ fusion at $10^{5} \mathrm{~K}$ and $10^{7} \mathrm{~K}$ respectively. The first vertical line, marks the maximal value at $10^{5} \mathrm{~K}$ and corresponds to $E=0.536 \mathrm{MeV}$, the second to $E=11.56$ $\mathrm{MeV}$ at $10^{7} \mathrm{~K}$.

the atomic numbers, and a coefficient given in $\mathrm{MeV}$. The constant that is represented numerically corresponds to $e^{2} / r_{0}$ where $e^{2} 2 \pi / h c=1 / 137.08$ and the nuclear radius is taken as $R=r_{0} A^{1 / 3}$. The $r_{0}$ to be taken can be very variable, there is no consensus value but it is typically the size of the radius of a nucleon. For example, under the assumption that Coulomb interaction plays the main role among all available electromagnetic forces potential associated with the $\mathrm{U}^{238}$ atoms is $V \approx 700 \mathrm{MeV}$, or temperatures of the order of $\approx 10^{12} \mathrm{~K}$.

Classically, the probability of achieving the fusion at temperatures like the sun falls exponentially due to the Boltzmann distribution, so it will be in the order of $\sim e^{-33245} \sim 0$.

Quantum-mechanical tunneling allows the protons to go through the Coulomb barrier with a probability that is much larger than this, though still exponentially suppressed. Radiating with an intense electromagnetic field will deform the potential barrier and thus enhance or suppress tunneling [1]. In astrophysical objects, like stars, the probability for a thermonuclear reaction to occur depends mainly on two factors. The first factor is the velocity distribution of the nuclei in the plasma and is usually given by a Maxwell-Boltzmann distribution. The second factor is the nuclear reaction cross section and contains the tunneling probability through the Coulomb barrier. Most thermonuclear reactions in stars take place at neither very low energies where the reaction cross section small, nor at relatively high energies where there a few number of nuclei. Nuclear reactions in a stellar plasma will occur near energies where the product of velocity distribution and cross section reaches at maximum.

The thermonuclear rate per particle pair for a reaction involving two nuclei is given by, [4], [6]:

$$
\langle\sigma\rangle=\sqrt{\frac{8}{\pi m}} \frac{1}{(K T)^{3 / 2}} \int_{0}^{\infty} E \sigma(E) e^{-E / K T} d E
$$

where $\sigma(E)$ is the cross section of the nuclear reaction at a certain energy $E$, energy of the center of mass. For a nonresonant reaction it can be factored into a part describing the strongly energy dependent tunneling probability through the Coulomb barrier and a part representing the weakly energy- 
dependent properties of the nuclear interior of the system, as $\sigma(E)=E^{-1} e^{-2 \pi \beta \sqrt{1 / E}}$.

The rate per particle is given then by:

$$
\langle\sigma\rangle=\frac{\sqrt{\frac{8}{\pi m}}}{(K T)^{3 / 2}} \int_{0}^{\infty} e^{-2 \pi \beta \sqrt{1 / E}} e^{-E / K T} d E
$$

where we have ignored the astrophysical factor $S(E)$, which is assumed to vary very slowly with the energy [4].

The major contribution in the above integral comes from the exponential terms, from this we can obtain the maximum value for a cross section at a given temperature. Optimizing the function $e^{-2 \pi \beta \sqrt{1 / E}} e^{-E / K T}$, we obtain the maximum value for $E$ :

$$
E_{o}=(\pi K T \beta)^{2 / 3}
$$

where $\beta \approx \alpha Z_{1} Z_{2} \sqrt{\mu / 2}$. This maximum value is called the Gamow peak, in $\mathrm{MeV}$ is located at [7]:

$$
E_{o}=0.1220\left(Z_{1}^{2} Z_{2}^{2} \frac{M_{1} M_{2}}{M_{1}+M_{2}}\left(\frac{T}{10^{9}}\right)^{2}\right)^{1 / 3}
$$

and the region $E_{o} \pm \Delta E_{o} / 2$ represents the effective energy for a nuclear non-resonant reaction, with

$$
\Delta E_{o}=0.2368\left(Z_{1}^{2} Z_{2}^{2} \frac{M_{1} M_{2}}{M_{1}+M_{2}}\left(\frac{T}{10^{9}}\right)^{5}\right)^{1 / 6} .
$$

In the case of $\mathrm{U}^{238}$ at $10^{7} \mathrm{~K}$ this value is $E_{o}=11.568 \pm 0.230$ $\mathrm{MeV}$. The dependence in the temperature open different regions suitable for fusion. At $10^{5} \mathrm{~K}, E_{o}=0.536 \pm 0.004$ $\mathrm{MeV}$. It can be seen that although to lower temperatures the energy for fusion is smaller, the window closes very rapidly. In the Fig. 2 is sketched the cross section for an uranium-uranium fusion, where it seems to stay stable after around $1.5 \mathrm{MeV}$.

\section{Modified Assisted Fusion by X-Ray Free ELECTRON LASER ENHANCEMENT}

The tunneling probability is the key component to achieve fusion. The potential barrier that has to be overcome, and the energies associated are too large. The Sauter-Schwinger effect predicts the creation of electron-positron pairs from the vacuum due to a quasi-constant strong electric field. The paircreation can be exponentially enhanced without destroying the tunneling nature of this mechanism by adding a weaker temporal Sauter pulse, a weak field, with a frequency above a certain threshold. Apart from the deformation of the potential barrier, the time dependence plays a crucial role for assisting tunneling through the Coulomb barrier, in close analogy to the dynamically assisted Sauter-Schwinger effect. In this original form of the dynamically assisted Sauter-Schwinger effect, the critical frequency is independent of the ratio of the relevant fields. Using the lower order of a Floquet ansatz, fields with wavelengths $\leq 50 \mathrm{pm}$ gives a dynamically assisted probability of tunneling,

$$
P \sim \exp \left\{-\pi \sqrt{\frac{2 \mu c^{2}}{E+\frac{h}{2 \pi} \omega}} \alpha\right\}
$$

and a prefactor that scales with the intensity of the incident field. With a reduced mass for two uranium atoms $\mu c^{2} \sim$ $112.2 \mathrm{GeV}$, an initial energy of $E \sim 1 \mathrm{KeV}$ and $\frac{h}{2 \pi} \omega \sim 10 \mathrm{KeV}$, the probability exponent is of the order of $P \sim 10^{-45}$. This is called the Floquet approach.

A more involved WKB scheme is presented [1], employing an ansatz $\psi=C e^{i S}$ we find an equation for $S(t, r)$, the eikonal equation, which can be separated into a zeroth order term $S_{0}(t, r)$ for the static tunneling and corrections $S_{1}(t, r)$ induced by the electromagnetic field. The tunneling assistance is highly dependent of the pulse profile in time. For energies below the potential barrier, tunneling regime, the $S_{1}(t, r)$ can be approximated by

$$
S_{1} / \frac{h}{2 \pi} \approx W(t) e^{\frac{h}{2 \pi} \omega \frac{\pi}{4}} \sqrt{\frac{2 \mu}{\epsilon^{3}} \alpha}
$$

where $W(t)=\frac{i q_{e f f} e^{i \omega t} \epsilon^{2}}{2 \mu \alpha\left(\frac{h}{2 \pi} \omega\right)^{2}}$ and $q_{e f f}=q \Delta m / m_{T}$ is the effective charge, given by the mass difference $\Delta m$ and the total mass $m_{T}$. Considering a sinusoidal pulse dependence, probability has a contribution of both orders, as

$$
\left|e^{i S_{0} / \frac{h}{2 \pi}+i S_{1} / \frac{h}{2 \pi}}\right|^{2} \sim e^{-2\left|S_{0}\right|} \mathcal{J}_{o}\left(2\left|S_{1}\right| / \frac{h}{2 \pi}\right)
$$

averaged in time, where $\mathcal{J}_{0}$ is the modified Bessel function of the first kind. For a fixed value of $S_{0}$ (static approach) the modulation is controlled by the Bessel function. The resulting probability will enhance as the $\mathrm{WKb} S_{1}$ decreases. The wavelengths of XFEL are $\geq 50 \mathrm{pm}$, which are much larger than the associated wavelengths of the initial kinetic energies estimated (that are around $1.4 \mathrm{pm}$ and $140 \mathrm{fm}$ ), so the vector potential can be approximated by a purely time dependent field $\vec{A}(t)$ [1]. The center-of-mass motion decouples from the relative coordinate $r_{-}=r_{D}-r_{T}$, with a dynamics governed by,

$$
L_{-}=\frac{\mu}{2} \dot{r}_{-}^{2}-V\left(\left|r_{-}\right|\right)+q_{e} \dot{r}_{-} \cdot A(t) .
$$

where $q_{e}=q\left(m_{1}-m_{2}\right) /\left(m_{1}+m_{2}\right)$ is an effective charge and the reduced mass is $\mu=1 /\left(m_{1}^{-1}+m_{2}^{-1}\right)$. If we consider the fusion of two atoms of the same species using this scheme, like ${ }^{238} \mathrm{U}$ atoms, $q_{\text {eff }}$ is zero, removing the electromagnetic contribution. To avoid this, we must include the spatial dependence of the ZFEL field.

In that case, the center of mass system will lead to

$$
L_{-}=\frac{\mu}{2} \dot{r}_{-}^{2}-V\left(\left|r_{-}\right|\right)+q \dot{r}_{-} \cdot A_{e}\left(t, r_{-}\right)
$$

where

$$
A_{e}\left(t, r_{-}\right)=\frac{m_{2} A\left(t, a_{1} r_{-}\right)-m_{1} A\left(t, a_{2} r_{-}\right)}{m_{1}+m_{2}}
$$

is an effective vector potential field, with $a_{1}=m_{2} /\left(m_{1}+m_{2}\right)$ and $a_{2}=-m_{1} /\left(m_{1}+m_{2}\right)$. The treatment in this case will involve integration and dependence on the shape of 

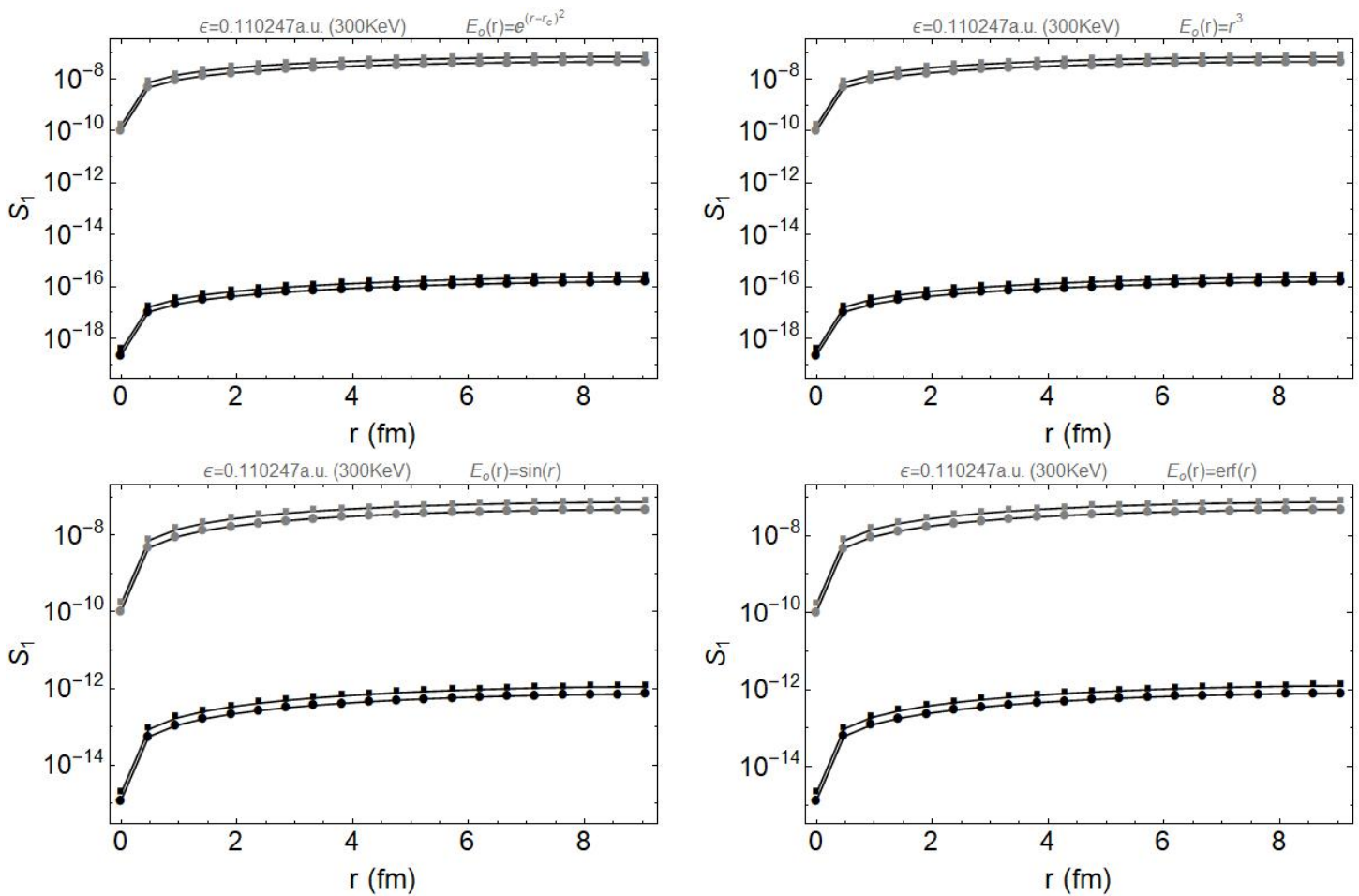

Fig. 3. WKB exponent $S_{1}$ for Deuterium-Deuterium fusion integrated in a pulse duration of $6 \mathrm{ps}$ and $\varepsilon=300 \mathrm{KeV}$. Black and gray markers corresponds to the different models, black markers for $r$ dependent fields and gray for only $t$ dependent fields. The values of $\frac{h}{2 \pi} \omega$ are $900 \mathrm{KeV}$ for squares and $60 \mathrm{KeV}$ for circles.

the incident radiation. If $m_{1}=m_{2}$, then $A_{e}\left(t, r_{-}\right)=$ $\left(A\left(t, r_{-} / 2\right)-A\left(t,-r_{-} / 2\right)\right) / 2$. Two cases are considered, if $A(t, r)$ is an even function of $r$, then the previous definition does not work, since it vanishes trivially. The second case when $A(t, r)$ is an odd function of $r$, where we obtain a finite contribution, that will take the general form,

$$
A_{e}\left(t, r_{-}\right)=A\left(t, r_{-} / 2\right) \text {. }
$$

Let us redefine the coordinates $r_{-}$such as,

$$
L=\frac{\mu}{2} \dot{r}^{2}-V(|r|)+q \dot{r} \cdot A(t, r / 2)
$$

Assuming that the potential between the atoms is given by a spatial scale $a= \pm 1 / 2$ given that the two species have the same mass.

As a first approach let's consider an odd vector potential in cylindrical coordinates, written as $A(t, r)=A(t)\{r, 0\}$. Employing the WKB ansatz $\Psi=e^{i S / \frac{h}{2 \pi}}$, we obtain the Hamilton-Jacobi equations,

$$
\partial_{t} S(t, r)+\frac{\left(\partial_{r} S(r, t)\right)^{2}}{2 \mu}+V(r)=0
$$

Following [1], we split into a static and non-static solutions $S_{0}$ and $S_{1}$ respectively, after linearizing in $A$ and $S_{1}$ leads to the set of equations,

$$
\begin{aligned}
& \partial_{t} S_{0}+\left(\partial_{r} S_{0}\right)^{2} / \mu+V(r)=0 \\
& \left(\partial_{t}+\frac{\partial_{r} S_{0}}{\mu} \partial_{r}\right) S_{1}+\left(\frac{q}{\mu} \partial_{r} S_{0}\right) A(t, r / 2)+ \\
& +q \partial_{t} \int d r A(t, r / 2) \approx 0 .
\end{aligned}
$$

where $\partial_{t} S_{0}=-\varepsilon$. The above system varies with respect to [1] in the extra term due to the spatial dependence of the pulse. The Eq (15) can be solved for a given form of the incident pulse. Let us consider a time dependent Gaussian pulse with a position dependent coefficient, given by $E(r, t)=\dot{A}(r, t)=$ $E_{0}(r) e^{-(\omega t)^{2}}$. From (14) and (15) we find the explicit the form,

$$
\begin{aligned}
& \left(\partial_{t}+\sqrt{\frac{(\varepsilon-V(r))}{\mu}} \partial_{r}\right) S_{1}+ \\
& +q \sqrt{\frac{(\varepsilon-V(r))}{\mu} \frac{\sqrt{\pi} \operatorname{erf}(\omega t)}{2 \omega} E_{0}(r)+} \\
& q e^{-(\omega t)^{2}} \int d r E_{0}(r) \approx 0
\end{aligned}
$$

where we considered a central collision of the nuclei along the $z$ axis, and assumed the maximum of the source term is at the turning point $r_{\varepsilon}$, and $V\left(r_{\varepsilon}\right)=\varepsilon$ [1].

\section{ObSeRVATIOnAl AND EXPerimental Perspectives FOR SUPER-HEAVY SPECIES}

Obtaining the data or detection of nuclear fusions fingerprints, could go into basically two different directions. The very first could be observations of extremely energetic astrophysical events, where looking for specific X-Ray emissions could lead to the possible detection. Another approach is to fuse elements with earth made technologies. We will give a small overview of what we think should be looked into both approaches along this section. 

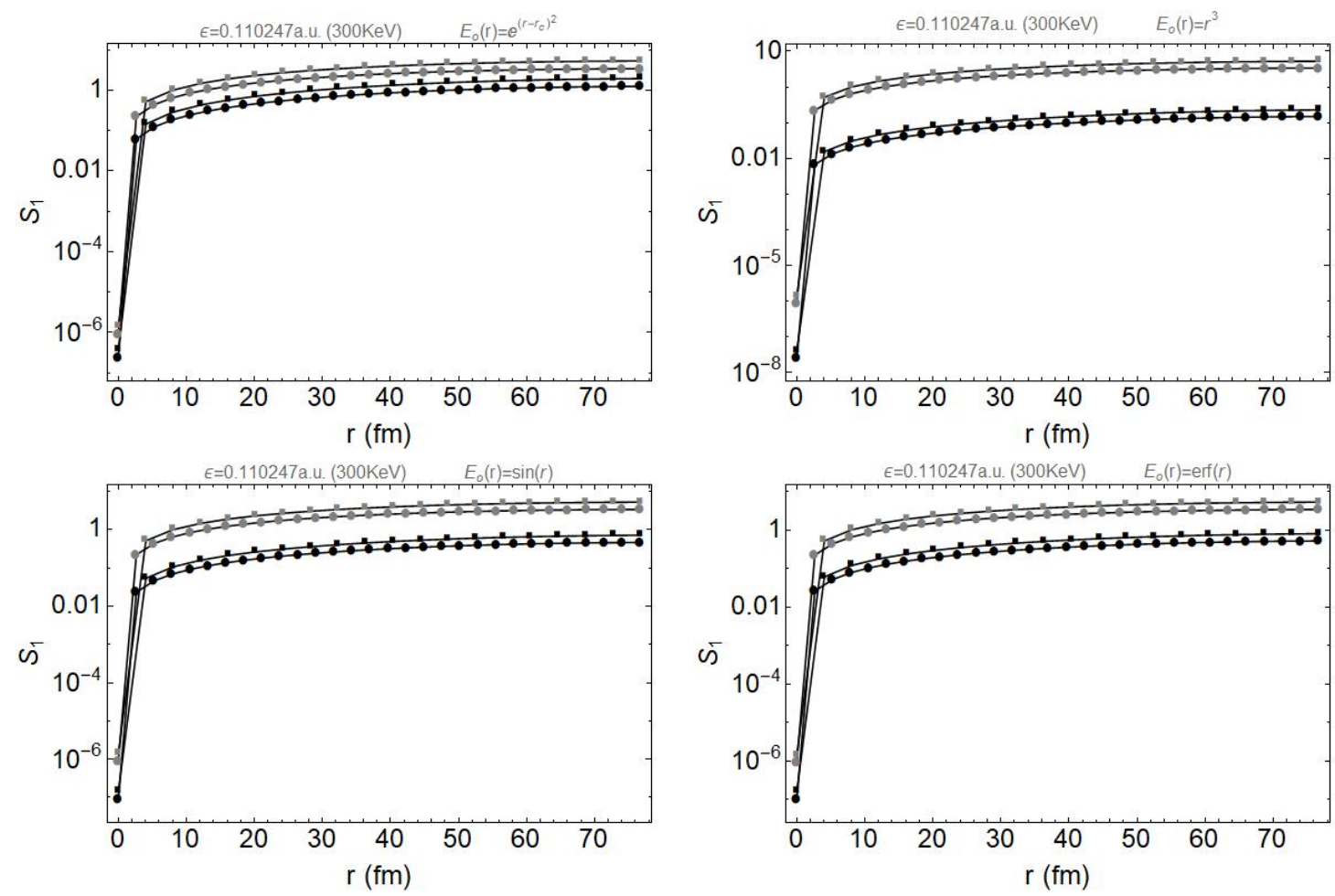

Fig. 4. WKB exponent $S_{1}$ for $\mathrm{U}^{238}$ fusion integrated in a pulse duration of $6 \mathrm{ps}$ and $\varepsilon=300 \mathrm{KeV}$. Black and gray markers corresponds to the different models, black markers for $r$ dependent fields and gray for only $t$ dependent fields. The values of $\frac{h}{2 \pi} \omega$ are $900 \mathrm{KeV}$ for squares and $60 \mathrm{KeV}$ for circles.
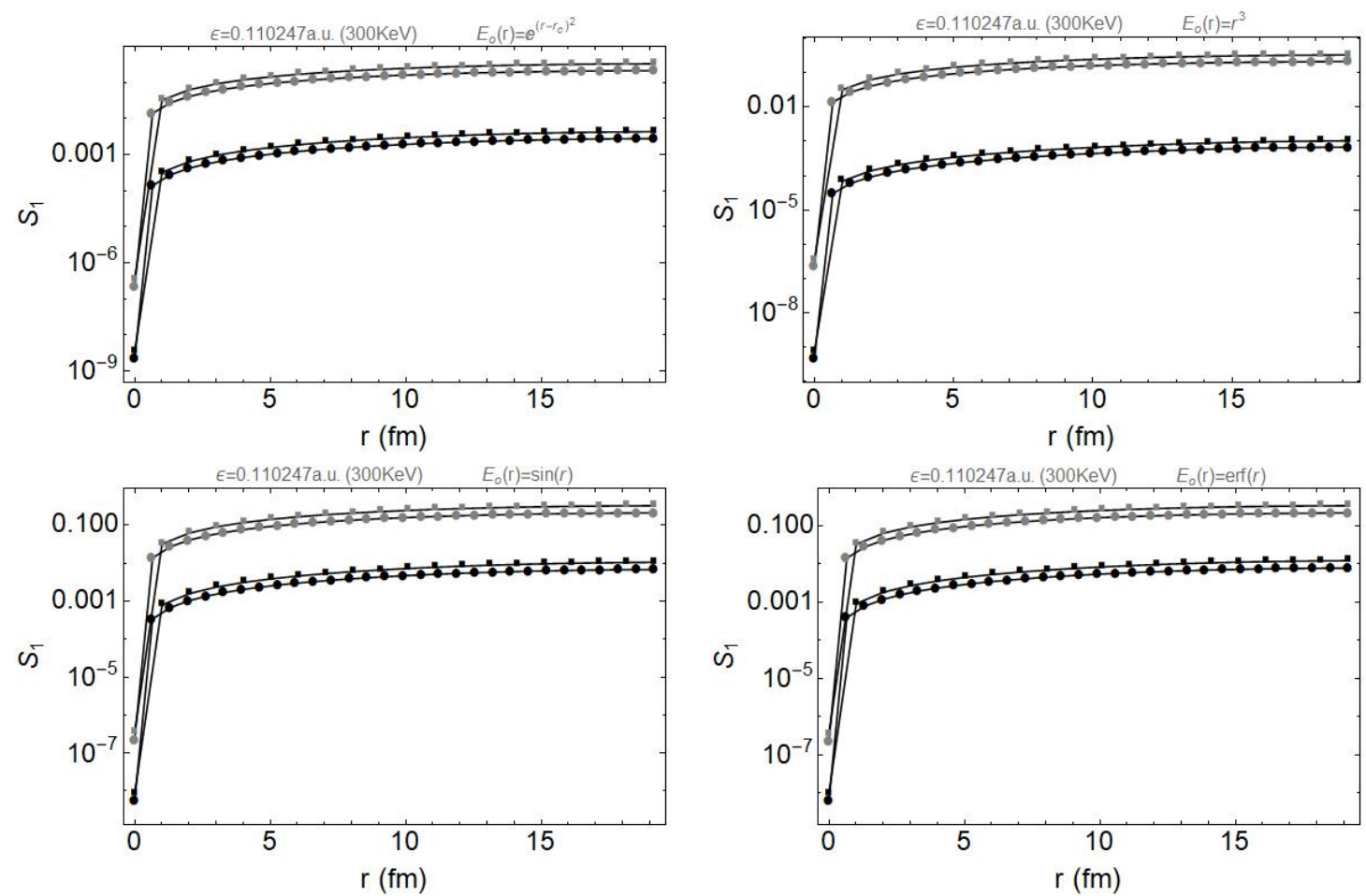

Fig. 5. WKB exponent $S_{1}$ for $\mathrm{Pd}^{106}$ fusion integrated in a pulse duration of $6 \mathrm{ps}$ and $\varepsilon=300 \mathrm{KeV}$. Black and gray markers corresponds to the different models, black markers for $r$ dependent fields and gray for only $t$ dependent fields. The values of $\frac{h}{2 \pi} \omega$ are $900 \mathrm{KeV}$ for squares and $60 \mathrm{KeV}$ for circles. 


\section{A. Expected X-Rays Emission Lines in Astrophysical Events}

The relative abundance of all chemical elements depends on their origin, the processes of nucleosynthesis. The lighter elements are believed to form in the hot big bang. However, from the Ytterbium its formation alternates between the slow process (s-process) in the interior of stars and the fast process (r-process) in neutron type star fusions. Slow neutron capture is called a s-process. This process occurs in an environment where the time between neutrons captured by the nuclei is longer than their half-life for beta decay. The s-process occurs in the final stage of life of stars, in their interior, where the number of free neutrons is low.

Nucleosynthesis by rapid neutron capture is called r-process. This process occurs in an environment rich in free neutrons where massive neutron capture by nuclei occurs. This process is very fast compared to their subsequent beta radioactive decay.

As it requires a very rich environment in free neutrons, it occurs in the waste ejected after neutron star fusions.(last sentence is not complete) These processes could be enhanced by the mechanisms already studied in previous sections. As a consequence, some evidence of super heavy elements would be expected as remnants of the nucleosynthesis processes; those, could emit specific radiations signatures depending on the electronic transitions involved.

As a consequence some evidence of super heavy elements would be expected as remnants of the nucleosynthesis processes; those, could emit specific radiations signatures depending on the electronic transitions involved. At first approximation based on hydrogen-like atoms, regarding the deformations due to large masses and relativistic correction could give us a clue about the remnants of some super heavy species abundances in the universe. A precise knowledge of electronic transition rates is necessary for the experimental identification of super heavy elements by their characteristic X-rays. The possible evidence based on proton-induced X-ray spectroscopy considering the $2 p^{3 / 2}$ level as final state has been investigated for several $Z$. There are transitions which can account for the measured energies but which can be missed either by too low transition rates or the presence of other dominant transitions around the same energy range.

At very high temperatures light elements such as hydrogen or helium has lost all their electrons. Only heavy elements can retain electronic structure in their innermost capacities. These elements are generally in a hydrogen state so the transitions between the inner layers can be described approximate and correspond to X-ray emissions.

The characteristic X-rays are the result of the Coulomb interaction between the incident electrons and the orbital electrons of the material (typically high atomic number $Z$ ) that constitutes the target. In terms of atomic physics, this process is considered collision loss.

When a Coulomb interaction event occurs between an incident electron and an orbital electron of the anode material, the energy transferred to the orbital electron is sufficient to eject it from the atomic shell; constituting what is known in atomic physics as an Auger electron, and consequently an electron of a higher energy level "falls" and occupies the

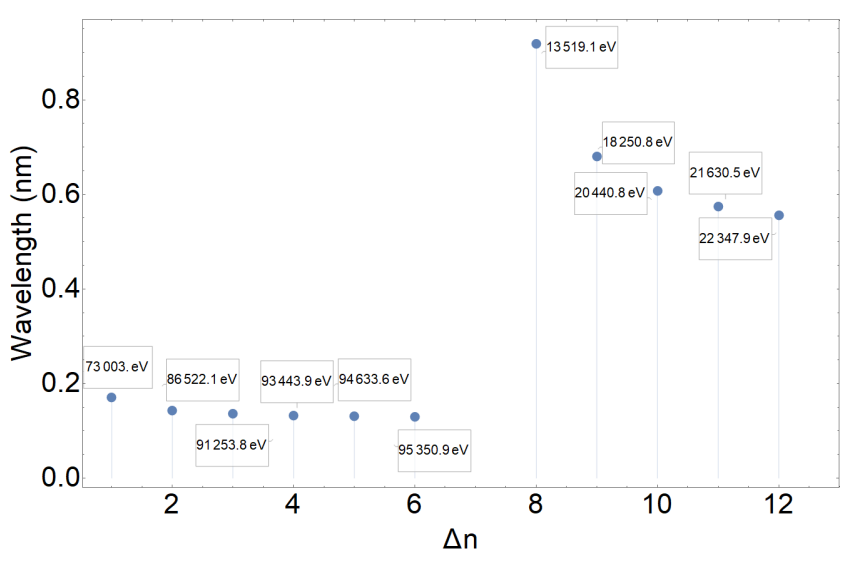

Fig. 6. X-Ray emission lines for $Z=115 . \mathrm{K}_{\alpha}$ and $\mathrm{K}_{\beta}$ transitions are shown.

created vacuum. The energy difference between the final and initial states (binding energies) (energy difference of the levels involved) is either emitted by the atom in the form of a characteristic photon (characteristic X-ray) or by transferring kinetic energy to the ejected orbital electron (Auger electron).

The photons emitted by electronic transitions between different atomic levels show a discrete distribution of energies in correspondence with each material. The energy of the photons emitted as characteristic X-rays can be estimated, according to the atomic levels involved, from the expression:

$$
E\left(n_{f}, n_{i}, Z\right)=\left(Z-C_{1,2}\right)^{2}\left(\frac{1}{n_{f}}-\frac{1}{n_{i}}\right) 13.6 \mathrm{eV}
$$

where $Z$ is the atomic number, $C_{1,2}$ are coefficients that represents the shielding effects. For a $\mathrm{K}_{\alpha}$ transitions the wavelength of a $Z=184$ atom would be around $10^{-2} \mathrm{~nm}$, beyond the $\mathrm{X}$ ray signature.

There are other sources of signatures for $Z=184$, its the mean positron energy is $\sim 200 \mathrm{keV}$, with the natural time scale for the unstable vacuum state $\sim 10-19 \mathrm{~s}$, which corresponds to an intrinsic resonance width of $<10 \mathrm{keV}$ [8]. Other positron emissions predominantly appear in nature as continuum distributions making this an specific characteristic to be looked for $Z=184$ although this can be lost with the collision formed supercritical charge.

The estimation of such events is weighted by an specific distribution of probabilities, in fact, for a D-T fusion the number of events expected in $1 \mathrm{~g}$ is of the order of $10^{23}$ reactions per unit of time, in contrast the relative probability for a ${ }^{238} \mathrm{U}$ fusion based on a enhanced fusion in a inertial confined setup, decreases by a factor of $10^{-14}$ in the case of a $r^{3}$ field. For such a $S_{1}$ the number of events for $1 \mathrm{~g}$ of ${ }^{238} \mathrm{U}$ will be approximately $\sim 10^{4}$. A significant dropping in the events reduces the intensity of the X-Rays. Roughly the luminosity is related to the available fuel mass in a start, as $\frac{L}{L_{\odot}}=\left(\frac{M}{M_{\odot}}\right)^{\alpha}$, where $\alpha$ depends of the specific object observed [9]. As an example, assuming that an astrophysical object lays on the main sequence, the correct relation is $\frac{L}{L_{\odot}} \approx 32000\left(\frac{M}{M_{\odot}}\right)$, so a reduction in fuel mass $M$ in $\sim 10^{19}$ significantly reduces de luminosity, making the observation of such events even more complex. 


\section{B. Laser Based Experimental Possibilities}

In this section we enumerate experiments that achieved promising characteristic energies to perform a fusion of superheavy elements.

Although the setups are conceived for light elements, the purpose is to review the foundational ideas as cornerstones for the type energies required to the proposed theoretical fusion. Recently in [10], it has been pointed out that alternatives to the current fusion approaches are leading in the direction of inertial confinement fusion.

The current techniques are based on the Tokamaks, a technology created with the sole purpose of generating heat excess. In these, a mixture of deuterium and tritium is heated to generate a plasma, as heating rises, the extreme collisions generate fusion that releases energy. The temperatures are extremely high and a continuous plasma confinement is required. Nonetheless, the energies are in the order of heavy fusion, albeit the reached time shots are very short. In order to synthesize super-heavy elements the beam intensities are above $10^{12}$ projectiles per second, which would result in burning the target and the detector. To overcome the Couloumb barrier for very heavy atoms "close" to ${ }^{238} \mathrm{U}$, center of mass equivalent energies in the other of $\sim 10 \mathrm{MeV}$, at minimal temperatures of $10^{7} \mathrm{~K}$ are needed, even in this scenario, cross section calculated from stellar non resonant reactions are very small.

Although tokamaks achieve high temperature and long confinement time, the density of gas in the reactor is low. Other devices confine plasma using magnets, but in a different arrangement, they achieve long confinement times but again still low densities. An extreme-high density and short confinement time approach is known as inertial confinement fusion (ICF).

\section{Internal Confinement Fusion}

In a nuclear fusion reaction it is essential to achieve adequate fuel confinement. There are two types of confinements, on the one hand the magnetic confinement (FCM), used by the European ITER and achieved using external magnetic fields and on the other hand the inertial confinement (FCI), used by the NIF in the United States.

The inertial confinement tries to achieve the right conditions for thermonuclear ignition during brief tenths of a nanosecond. For this it is basic to use two elements: a laser beam and the nuclear fuel. A hollow sphere containing deuterium-tritium (DT) is used as the nuclear fuel. The capsule has three layers. The outermost is a plastic form of $\mathrm{CH}$. The intermediate contains frozen DT and the nucleus contains gaseous DT.

In the direct drive approach, the capsule is introduced into the cavity where the reaction will occur and is fired on with laser beams increasing the energy of the outer layer where a hot plasma envelope is created. Other plasma controlled approaches in open traps are possible [11]. This energy is transferred inside the capsule and an implosion of the DT occurs [12]. As the outer layer released energy the inner region is compressed. When the interior is compressed, the

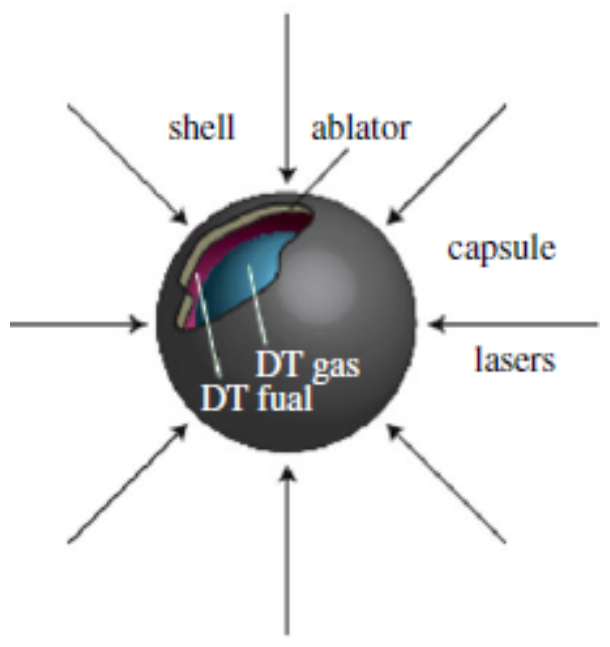

Fig. 7. A spherical capsule is prepared with a layer of DT fuel on its inside surface. As the capsule surface absorbs energy and ablates, pressure accelerates the shell of remaining ablator and DT fuel inwards-an implosion. By the time the shell is at approximately one-fifth of its initial radius it is travelling at a speed of many hundreds of kilometres per second. By the time the implosion reaches minimum radius, a hotspot of DT has formed, surrounded by colder and denser DT fuel.

kinetic energy of the interior particles is transformed into internal energy very quickly, causing a sudden increase in temperature in the central region, although not in the rest of the fuel. This reaction occurs only in the most central region, and the alpha particles created in the reaction and with little range, favor the reaction in the capsule nucleus. Those that manage to escape to the outer layer cause their energy to increase and thus, the reaction is also favored. During this period of time, known as the confinement time, the fuel increases its density, and the emitted radiation and neutrons are deposited on the walls of the chamber, their energy is absorbed and transferred to a cooling liquid. If the symmetry is sustained, the fuel will be compressed with a density one hundred times that of lead and a temperature of 50 million degrees Celsius. Under these conditions, a fusion burn should begin in a hotspot at the centre of and then propagate outwards consuming all the fuel. Another variant, known as fast ignition, uses two lasers: one to compress the capsule in the same manner as direct drive, and a second to shine a single beam at the compressed fuel and, with very short, very high-powered pulse, set it alight, see Fig 7. Different encapsulations should be needed for larger atoms, the alpha emission would be larger and the number of neutrons deposited during the confining time has to be estimated, due to an expected rapid fuel consumption.

\section{Fast Ignition Realization EXperiment (FIREX)}

Fast ignition (FI) is a promising approach for high-energygain inertial confinement fusion in the laboratory. Several experiments have been performed on the GEKKO-LFEX laser facility, at the Institute of Laser Engineering in Osaka University. 


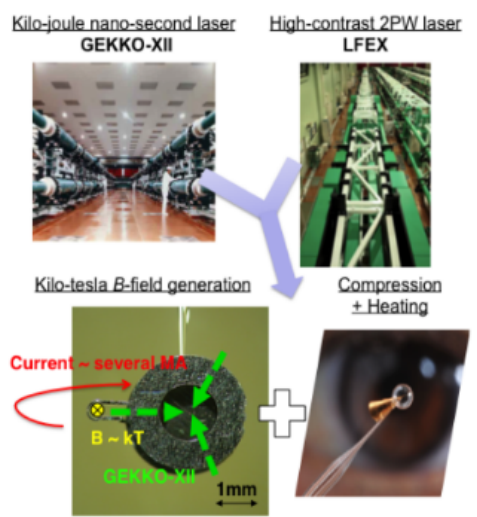

Fig. 8. A spherical fuel target is compressed by the GEKKO-XII laser beams and the compressed core is heated by the high-contrast $2 \mathrm{PW}$ laser. Kilo-tesla magnetic field is generated by a capacitor-coil target driven by part of the GEKKO laser.

The project that started in 2001, aims to achieve high ignition temperatures [13]. The experiment has been successfully implemented in other fields, like intense magnetic fields driven by lasers and plasma expansion [14], [15], [16]. In the fast ignition scheme, a high-intensity laser is converted into an energetic charged particle (electron/ion) beam through laserplasma interactions. The high-density plasma is heated by the energetic particle beam. The target consist in a $\mathrm{Cu}$ contained $\mathrm{CH}$ sphere and a parylene coating layer to protect the $\mathrm{Cu}$ atoms from the direct incident lasers. The GEKKO-XII is a set up of twelve lasers, of $526 \mathrm{~nm}$, with energies as much as 200J per beam. At this point it resembles the ICF approach. At the equatorial section of the sphere, a FLEX laser is injected, generating a forward moving fast electron beam [17]. See Fig. 9. During laser ablation, a shock is launched in the target, which moves inward until $2.6 \mathrm{~ns}$, forming a high-density shell surrounding the target center. The laser ablation produces a hot $(\sim 2 \mathrm{keV}$ or 23 millions $\mathrm{K}$, more than three thousand times the sun temperature) and large density gradient scale length. The energy gained by fast electrons is accounted by taking advantage of the fact that the fast electrons excite $\mathrm{Cu} \mathrm{K} \alpha$ emission when passing through the $\mathrm{Cu}$ contained plasma. In principle, as long as the plasma conditions and the cross-section are known, including its absolute intensity and spatial features, allows one to derive the information of the fast electron beam, which is usually characterized by an exponential distribution for the energy spectrum and by a Gaussian distribution for the angular divergence.

In [17] they found that the laser-to-core energy coupling efficiency could be improved by optimizing the implosion and super-penetration performances, by adding an channel pulse. They expect larger than $12 \%$ of efficiency in laser to core energy coupling but with a simpler set up than other known experiments [18]. Similar experiments and achieved equally promising results. In [19] they report ion acceleration by highcontrast in ps pulses. They simultaneously deliver four laser beams with a FWHM pulse duration of 1.5 ps, see Fig. 10 . The maximun laser energy in total is $1 \mathrm{~kJ}$ on the target. A long laser beam duration is reported, achieved by adjusting the laser

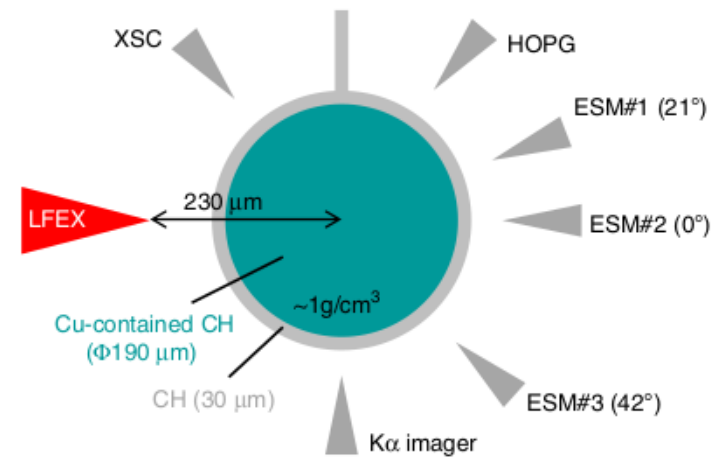

Fig. 9. Experimental setup, laser power and areal density.

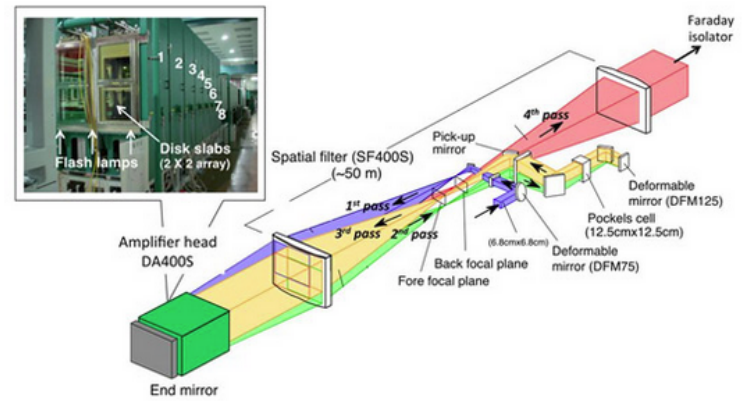

Fig. 10. Configuration of the main amplification section of LFEX.

path length of the OAP,, setting intervals of 1.5 ps between the four pulses, for instance,they obtain an extended pulse of $6 \mathrm{ps}$ (FWHM) as the longest case using the four pulses. Electron temperatures of $0.41,1.13$ and $0.97 \mathrm{MeV}$ for $1.5,3$ and $6 \mathrm{ps}$, respectively are reported.

\section{E. Ultra-Short Laser Pulses in Non-Thermal Plasma Condi- tions}

Laser fusion has been studied mostly by heating of the fuel under conditions of thermal equilibrium. Several measurements were known related to the laser interaction with nuclear fusion fuel, mostly with deuterium or mixed with tritium. For fusion, $\mathrm{T}$ has to be around or far above 50 Million degrees Celsius, which is how pressure is usually given in plasma. The use of sub-picosecond, chirped pulse amplification (CPA) laser pulses produce energy densities for generation of nonthermal, nonlinear-force driven pressures that are nine orders of magnitude temperatures than those needed for fusion energy in thermal equilibrium conditions. Although the ignition has been developed for Hydrogen-Boron fusion, its enhancement could be in principle extrapolated to realize new heavy elements fusion.

The basic principle relies on the non linearity of the electromagnetic field and its very high intensity. A laser is driven into a sample, the laser contributions are highly non linear depending on the intensity and the pulse frequency, see Fig. 11 , this produces a non linear force that drives a plasma block towards the laser beam position and another plasma block in 


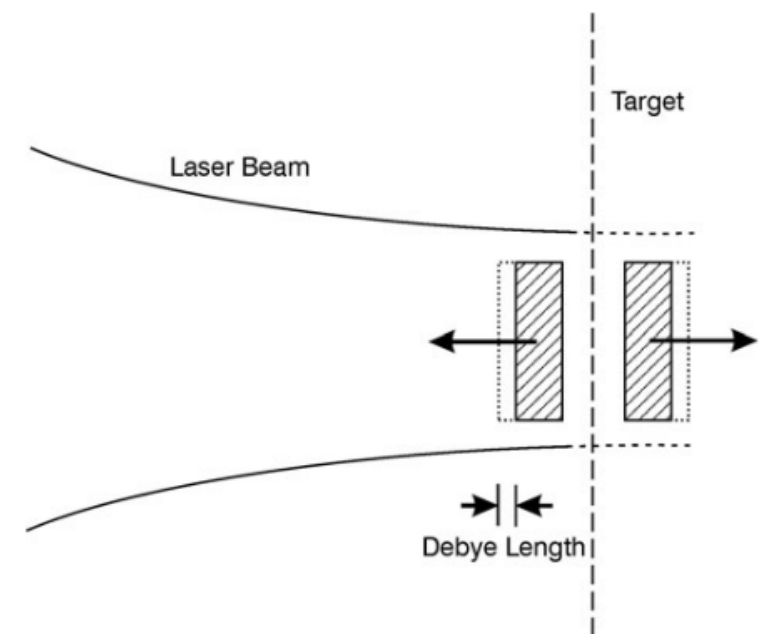

Fig. 11. Non-linear incident laser beam. The gradient of the non linear effects due to the electromagnetic field induces acceleration towards the laser and inside the target. Each block has a frontal cloud of electrons of the order of the Debye length.

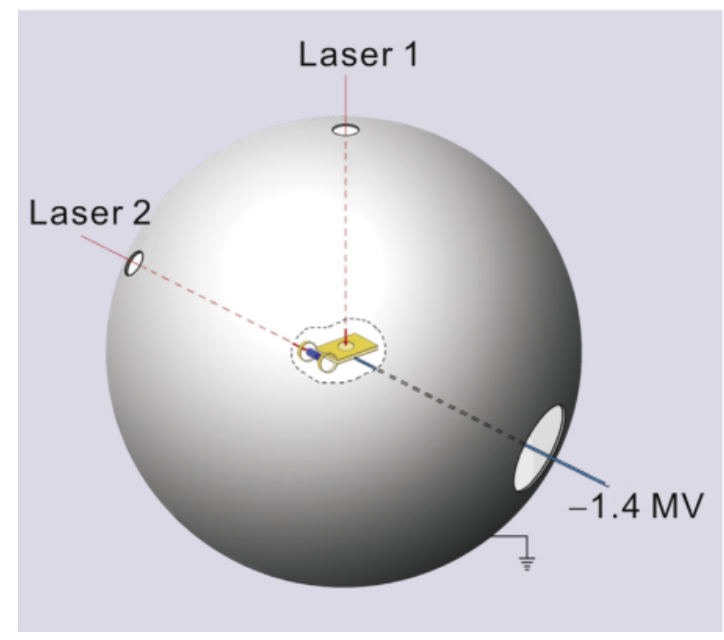

Fig. 12. Two incident laser beams. Laser 1 produces a strong magnetic field in the capacitor-coil reaction unit in the center of the sphere and laser pulse 2 is use to initiate the non-thermal non-linear force-driven reaction fuel cylinder

the direction of the target [20], [21], [22]. The equation of motion of the force density $\mathbf{f}$ in a plasma, given by the thermokinetic pressure $p$, the particle density $n$ and the temperature $T$ is given by,

$$
\mathbf{f}=-\nabla p+\mathbf{f}_{\mathrm{NL}}=-\nabla \mathbf{p}-\nabla \cdot \mathbf{M}
$$

where $\mathbf{M}$ is the Maxwell's stress tensor of the electric and magnetic fields $\mathbf{E}$ and $\mathbf{H}$ of the laser [20], [22]. To optimize the heat, by using non equilibrium plasma or non-local thermal equilibrium (non-LTE), is needed that the term due to non linear forces $\mathbf{f}_{\mathrm{NL}}$ can produce much higher pressures than the thermal pressure $p$. The non linear force drives to produce highly energetic collisions, reaching temperatures of several order of magnitude larger than usual thermal equilibrium approaches. The avalanche effect to trigger the plasma is produced and modulated with another laser, and it is trapped into a magnetic coil trap [21]. See Fig. 12. In the experiment reported in [20] for the clean HB11 fusion reaction the classical fusion energy gains are increased by more than nine orders of magnitude.

\section{Discussion And Results}

We analyzed qualitatively solutions of the WBK exponent $S_{1}$ integrating on pulses of $6 \mathrm{ps}$ and limited to distances up to the classical turning point. The field energies used $\frac{h}{2 \pi} \omega \sim \varepsilon / 5$ $\mathrm{KeV}$ and $\frac{h}{2 \pi} \omega \sim 3 \varepsilon \mathrm{KeV}$ and initial kinetic energy $\varepsilon \sim 100$ $\mathrm{KeV}$, based on possible experimental setups in [19].

For the sake of simplicity in the calculations and reducing the numerical results cost, we use atomic units, where $\frac{h}{2 \pi}=c=$ $e=m_{p}=m_{n}=k_{B}=k_{c}=1$, the results are expressed in a.u. (atomic units), for instance $\frac{h}{2 \pi} \omega \sim \varepsilon / 5 \mathrm{KeV}=\frac{h}{2 \pi} \omega=$ 0.022049 a.u., $\frac{h}{2 \pi} \omega \sim 3 \varepsilon \mathrm{KeV}=\frac{h}{2 \pi} \omega=0.330741$ a.u. and $\varepsilon=300 \mathrm{KeV}=0.110247$ a.u.(Hartrees).

Following [1], $E(r, t)=\dot{A}(r, t)$, is a Gaussian, i.e.

$$
A(t, r / 2)=\frac{\sqrt{\pi} E_{0}(r) \operatorname{erf}(\omega t)}{2 \omega}
$$

with $\operatorname{erf}(z)$ the error function.

To include the position dependence on the radiation field the function $E_{0}(r)$ must be a non even function of $r$, which can be achieved by an asymmetric distribution of the incident laser field. We used simple spatial distributions functions to test the model performance, namely $\sin (r), \operatorname{erf}(r), r^{3}$ and $e^{-\left(r-r_{\epsilon}\right)^{2}}$. In Fig. 4 and Fig. 3 we show the $S_{1}$ coefficient obtained for each field spatial distribution, compared with the same scenario without spatial dependence in the, $A=A(t)$. Each dot represents an integration in $6 \mathrm{ps}$ and from $r=0$ to the classical turning point $r=r_{\epsilon}$. There is a clear enhancement with respect to space independent situation. In Fig. 3 we show the Deuterium-Deuterium (D-D) assisted fusion WKB exponent behavior with respect to the separation between the nuclei.

A comparison between the models reveals that the relative enhancement is closely independent of the chosen spatial function $E_{0}(r)$. For $\frac{h}{2 \pi} \omega=900 \mathrm{KeV}$ and $\frac{h}{2 \pi} \omega=60 \mathrm{KeV}$ there is a reduced additional enhancement relative to the gap between time and space dependent is the leading effect, see Fig. 3. The difference in magnitude between both approaches is preserved along the distancing, reaching constant values as both nucleus are more apart. The order of magnitude $S_{1}$ grows rapidly around $\approx 0.5 \mathrm{fm}$ between the nucleus for both models. Both models reach a top value near $\approx 8 \mathrm{fm}$. The $S_{1}$ on those regions is on the order of $\approx 10^{-8}$ for a space independent field and $S_{1} \approx 10^{-17}$ for the exponential and $r^{3}$ test fields, an increase in nine orders of magnitude.

In the case of the sin and erf test fields, the enhancement is around $10^{-13}$, at lest four orders of magnitude below the previous enhancement, but still five orders of magnitude above the $r$ independent field model.

We tested our model for $\mathrm{U}^{238}$, the stablest heavy element available, with the same $E_{0}(r)$ functions as seen in Fig. 4. For this, the model is highly dependent of the incident laser test field. For an exponential, sin and erf functions the difference between the models is less accentuated, no more than one order of magnitude. The $r^{3}$ field reports a better enhancement of $\approx 10^{-2}$ above the $r$ independent approach. 
Palladium $\mathrm{Pd}^{106}$ is tested as an intermediate species. The results shows a similar behavior to $\mathrm{U}^{238}$, see Fig. 5. The $r^{3}$ spatial shape has the leading enhancement in the $S_{1}$ exponent.

These results suggest that shape if incidence of the laser on heavy species confined fusion setup has a remarkable effect. The asymmetry respect to the incident laser onto each atom increases the Sauter effect described in [1].

The probability of the ${ }^{238} \mathrm{U}$ relative to the D-D fusion assisted with a Sauter effect reduces to only $\approx 10^{-14}$ according to the WKB exponent enhancement.

From the experimental point of view, a setup that reaches kinetics energies of the order of $\approx 300 \mathrm{KeV}$ with a close to $6 p s$ incident Sauter pulse, and working with frequencies equivalents to $\approx 60-900 \mathrm{KeV}$, we estimate a relative probability of fusion for ${ }^{238} \mathrm{U}$ of $10^{-14}$, a clear boost in the associated probability compared to the classical approaches to such heavy atoms.

This kind of boost in the WKB exponent at least conceptually suggest a starting point to fuse super-heavy species in an inertial confinement setup, assisted by electromagnetic pulses of large energy. The understanding of the tunneling is far for complete, even the addition of minor improvements changes dramatically the physical outcome, boosting the motivation for further studies.

\section{CONCLUSION}

The extreme Coulomb forces, and the effect of shells makes the nuclear binding energy in the ground state increase, a fission barrier arises, and strongly increased stability of the heaviest nuclei, leading to the existence of a large region of superheavy elements suitable to be synthesized.

To achieve fusion the Coulomb repulsion between the nuclei has to be overcome, because of their proximity the energy associated with this barrier is too large. Quantum-mechanical tunneling allows protons to go undergo the Coulomb barrier with larger probabilities, though still exponentially suppressed. The tunneling probability is the key component to achieve fusion. Adding a weaker temporal Sauter pulse, with a frequency above a certain threshold, pair-creation can be exponentially enhanced in the region of fusion without destroying the tunneling nature, then dynamically assisted Sauter-Schwinger effect appears, the critical frequency is an independent strength relation between the fields. Using the lower order of a Floquet ansatz, fields with wavelengths $\leq 50 \mathrm{pm}$ gives a dynamically assisted probability of tunneling. With a reduced mass for two uranium atoms $\mu c^{2} \sim 112.2 \mathrm{GeV}$, an initial energy of $300 \mathrm{KeV}$ and $\frac{h}{2 \pi} \omega \sim 60-300 \mathrm{KeV}$, the probability exponent is enhanced highly. Using perturbation theory, the WKB exponent can be calculated, considering a radiation field that in general is time and space dependent, the solutions are split into $S_{0}$ and $S_{1}$, as zero and first order correction respectively.

Numerical solutions for $S_{1}$, base on pulses up to $6 \mathrm{ps}$, that are achievable in recent experiments, we obtain large enhancements depending on the $E_{0}(r)$ spatial function of the field. A handful of experiments could reach the desire conditions, at least to a hypothetical degree, the European ITER and Fast Ignition Realization EXperiment (FIREX). Fast Ignition experiments, like GEKKO-XII achieve ion acceleration by high-contrast in ps pulses. They simultaneously deliver laser beams with a pulse duration of $1.5 \mathrm{ps}$. The maximum laser energy in total is $1 \mathrm{~kJ}$ on the target. Adjusting the laser path length, setting intervals of $1.5 \mathrm{ps}$ between the pulses they obtain an extended pulse of 6 ps (FWHM) which increases the possible $S_{1}$ values.

Perspectives - In contrast to perturbation theory, the semiclassical approximation is not related to the smallness of the interaction which suits for problems of heavy atoms in the regime of inertial fusion and fast ignition. The next step is use a modified perturbation theory, with which it is possible to obtain asymptotic expressions for the two-center quasiradial and quasiangular wave functions for large inter-nuclear distances as shown in [23]. It is shown that in each order of $1 / R$, corrections to the wave functions are expressed in terms of a finite number of Coulomb functions with a modified charge. The mentioned method ensures simple uniform estimates for eigenfunctions at arbitrary large inter-nuclear distances $R$, including $R \leq 1$.

\section{ACKNOWLEDGMENT}

NB and IV want to thanks Dr. Rafael Martin Landrove and Dr. David Verrilli for their contributions and insightful observations in the preparation of the manuscript.

\section{REFERENCES}

[1] F. Queisser and R. Schützhold, "Dynamically assisted nuclear fusion," Phys. Rev. C, vol. 100, p. 041601, 2019.

[2] F. Tennie, V. Vedral, and C. Schilling, "Universal upper bounds on the bose-einstein condensate and the hubbard star," Phys. Rev. B, vol. 96, p. 064502, 2017.

[3] K. P. Schmidt, J. Dorier, A. Läuchli, and F. Mila, "Single-particle versus pair condensation of hard-core bosons with correlated hopping," Phys. Rev. B, vol. 74, p. $174508,2006$.

[4] J. R. Newton, C. Iliadis, A. E. Champagne, A. Coc, Y. Parpottas, and C. Ugalde, "Gamow peak in thermonuclear reactions at high temperatures," Phys. Rev. C, vol. 75, p. 045801, 2007.

[5] A. Stafano and M. Jürgen, Physics of Inertial Fusion: Beam Plasma Interaction, Hydrodynamics, Hot Dense Matter. OUP Oxford, 2004.

[6] N. Bathia, S. S. Malik, and A. K. Jain, "Fusion near the coulomb barrier for the synthesis of heavy and superheavy elements: A theoretical approach," Eur. Phys. J. A, vol. 26, pp. 241-251, 2005.

[7] J. Humblet, W. Fowler, and B. A. Zimmerman, "Approximate penetration factors for nuclear reactions of astrophysical interest," Astron. Astrophys., vol. 177, pp. 317-325, 1987.

[8] J. Reinhardt et al., "Treatise on heavy ion science," High-Energy Atomic Physics, vol. 5, 1985.

[9] N. Duric, Advanced astrophysics. Cambridge University Press, 2003.

[10] D. Clery, "Alternatives to tokamaks: a faster-better-cheaper route to fusion energy?" Philosophical Transactions of the Royal Society A: Mathematical, Physical and Engineering Sciences, vol. 377, p. 20170431, 2019.

[11] D.V.Yakovlev, A. Shalashov, E. Gospodchikov, V. Maximov, V. Prikhodko, V. Savkin, E. Soldatkina, A. Solomakhin, and P. Bagryansky, "Stable confinement of high-electron-temperature plasmas in the GDT experiment," Nuclear Fusion, vol. 58, no. 9, p. 094001, 2018.

[12] S. Eliezer, A. Ravid, Z. Henis, N. Nissim, and J. Martinez Val, "Laserinduced fusion detonation wave," Laser and Particle Beams, vol. 34, no. 2, p. 343-351, 2016.

[13] H. Azechi, "A pathway to laser fusion energy in japan," J. Phys.: Conf. Ser., vol. 717, p. 012119, 2016. 
[14] Y. Abe, "Laboratory-produced quasi-static magnetic field with astronomical strength driven by ultra-high intensity lasers," in Proceedings of the Samahang Pisika ng Pilipinas, vol. 37, no. 1, 2019, pp. SPP2019-INV-3A-04.

[15] T. S. Natsumi Iwata, Yasuhiko Sentoku and K. Mima, "Plasma expansion accompanying superthermal electrons in over-picosecond relativistic laser-foil interactions," Plasma Physics and Controlled Fusion, vol. 62, no. 1, p. 014011, oct 2019.

[16] M. Ota, A. Morace, R. Kumar, S. Kambayashi, S. Egashira, M. Kanasaki, Y. Fukuda, and Y. Sakawa, "Collisionless electrostatic shock acceleration of proton using high intensity laser," High Energy Density Physics, vol. 33, p. 100697, 2019.

[17] T. Gong, H. Habara, K. Sumioka et al., "Direct observation of imploded core heating via fast electrons with super-penetration scheme." Nat. Commun., vol. 10, p. 5614, 2019.

[18] L. Jarrott, M. Wei, C. McGuffey et al., "Visualizing fast electron energy transport into laser-compressed high-density fast-ignition targets," Nature Phys., vol. 12, pp. 499-504, 2016.

[19] A. Yogo, K. Mima, N. Iwata et al., "Boosting laser-ion acceleration with multi-picosecond pulses," Sci Rep, vol. 7, p. 42451, 2017.

[20] H. Hora, S. Eliezer, G. H. Miley, J. Wang, Y. Xu, and N. Nissim, "Extreme laser pulses for non-thermal fusion ignition of hydrogen-boron for clean and low-cost energy," Laser and Particle Beams, vol. 36, no. 3 , p. $335-340,2018$

[21] H. Hora, S. Eliezer, N. Nissim, and P. Lalousis, "Non-thermal laser driven plasma-blocks for proton boron avalanche fusion as direct drive option," Matter and Radiation at Extremes, vol. 2, no. 4, pp. 177 - 189, 2017.

[22] H. Hora, "Nonlinear confining and deconfining forces associated with the interaction of laser radiation with plasma," The Physics of Fluids, vol. 12, no. 1, pp. 182-191, 1969.

[23] M. Hnatich, V. Khmara, V. Lazur et al., "The wkb method for the quantum mechanical two-coulomb-center problem." Theor Math Phys, vol. 190 , p. $345-358,2017$. 